Tableau II.

Fromage salé, vieux de 4 semaines . . . . . . . 5,1 . . . 5,05

$$
\begin{aligned}
& \mathrm{pH} \text { Col. } \mathrm{pH} \text { Elec. } \\
& 5,2 \ldots .5,12
\end{aligned}
$$

...... $5,1 \ldots 5,00$

Fromage non salé, vieux de 6 heures . . . . . . 5,3 . . . . 5,32

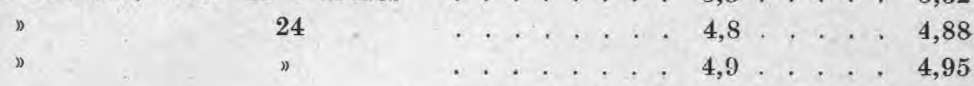

La composition des solutions-types est donnée dans le Tableau III, elle exige :

I. Solution de $\mathrm{NaOH}$ à $0,1 \mathrm{~N}$. ne contenant pas de carbonates.

II. Solution d'acide lactique à $0,1 \mathrm{~N}$.

Pour éliminer l'anhydride et le lactide de l'acide lactique, il faut chauffer la solution $\pm \mathrm{N}$. pendant plusieurs heures, à la température d'ébullition.

$\mathrm{Cm}^{3}$ acide lactique à $0,1 \mathrm{~N}$.
pour $200 \mathrm{~cm}^{3}$
40,3
40,6
41,0
41,5
42,0
43,0
44,0
45,2
48.0

\title{
TableaU III.
}

$\mathrm{Cm}^{3} \mathrm{NaOH}$ à $0,1 \mathrm{~N}$. pour $200 \mathrm{~cm}^{3}$

$\mathrm{pH}$

40

6,0

40

(Traduction de R. N. Göransson.)

\section{RECHERCHES SUR LA PASTEURISATION BASSE DU LAIT. UNE NOUVELLE RÉACTION POUR CONTROLER LA TEMPÉRATURE DE CHAUFFAGE}

par le Professe ur ORLA-JENSEN, $\mathrm{D}^{\mathrm{r}}$ en philosophie et $\mathrm{D}^{\mathrm{r}}$ ès seiences,

arec la collaboration de C. LE DOUS, JoHANne JACOBSEN et N. C. OTTE.

\section{(Suite)}

Dans les essais résumés dans le tableau XXXV, nous avons employé du sérum de lait cru comme addition. Dans un essai de'contrôle, dans lequel nous avons ajouté à l'eau les mêmes proportions de sérum cuit, nous n'avons naturellement pas eu de montée de crème, car l'agglutinine y était détruite. Le tableau XXXV confirme en outre notre observation antérieure que, dans le sérum qui n'est pas du tout étendu, on n'obțient presque pas de montée de crème (quand la matière grassse a été 
ajoutée sous la forme de crème centrifuge). L'effet nuisible des substances retardatrices semble par contre annihilé quand on l'étend avec seulement $2 / 10$ d'eau.

TABLEAU $X X X V$

\begin{tabular}{|c|c|c|c|c|c|c|c|}
\hline \multirow{2}{*}{\multicolumn{5}{|c|}{$\begin{array}{l}\text { Eau et sérum, l'un et lautra } \\
\text { avec } 4 \% \text { de mat. grasse }\end{array}$}} & \multicolumn{2}{|c|}{ Crème } & \multirow{2}{*}{ A } \\
\hline & & & & & non étendu & ét ndu & \\
\hline $10 / 10 \mathrm{e}$ & ean & +0 & sérunı & * & 0,5 & traces & \\
\hline $8 / 10$ & n & $+2 / 10$ & , & * & 16,0 & traces & \\
\hline $6 / 10$ & n & $+4 / 10$ & , & * & 22,0 & 5,0 & 0,2 \\
\hline $4 / 10$ & , & $+6 / 10$ & 0 & * & 23,0 & 33,0 & 1,4 \\
\hline $2 / 10$ & " & $+8 / 10$ & " & * & 27,0 & 26,0 & 1,0 \\
\hline 0 & $n$ & $+10 / 10$ & 1) & * & 1,5 & 37,0 & 24,7 \\
\hline
\end{tabular}

Aussitôt qu'on a la conviction que l'agglutinine est le facteur principal de la montée de la crème, on comprend pourquoi on détruit la faculté de montée de la crème plus rapidement par le chauffage dans le lait étendu que dans le lait non étendu, car le laít étendu ne contient que la moitié de l'agglutinine que contient le lait non étendu et il peut done être beaucoup plus sensible à une diminution supplémentaire de la proportion d'agglutinine. Des eșsais précédents nous savons que le rapport A descend généralement au-dessous de 1 quand le lait a été chauffé 30 minutes à $62^{\circ} \mathrm{C}$., et la question est maintenant de savoir si en étendant avec un pau moins d'eau, on ne pourrait pas obtenir que le changement ait lieu précisément là où il devrait avoir lieu, c'est-à-dire après 30 minutes de chauffage du lait à $63^{\circ} \mathrm{C}$. D'après ce que nous savons de la question, il doit pouvoir être possible, au point de vue théorique pur, par l'addition d'une proportion d'eau convenable, d'obtenir le changement à n'importe quel moment, si on se tient dans la limite des températures auxquelles l'agglutinine est détruite.

Les tableaux XXXVI et XXXVII donnent les résultats de quelques essais dans lesquels nous a vons étendu le lait avec des proportions diverses d'eau. Le rapport de mélange $10 / 0$ correspond à du lait non étendu et $5 / 5$ à notrelait étendu habituel. Comme lelait de Jerseyaise, pour les raisons déjà indiquées, donnerait une très grande différence pour $\mathrm{A}$ dans le lait cru et dans le lait chauffé, si nous mesurions la couche de crème après un court délai, nous l'avons aussi dans l'essai actuel mesurée après 1 heure $1 / 2$. Le résultat ne fut toutefois pas encourageant et fréquemment la couche de crème n'a pas pu se rassembler définitivement dans ce court laps de temps de sorte que par cette voie, on ne peut rien obtenir.

Nous voyons, en ce qui concerne d'abord le lait cru, que le degré de dilution influe étonnamment peu sur la quantité de crème. L'optimum pour le lait ordinaire est généralement très près du rapport de mélange 
TABLEAU XXXVI

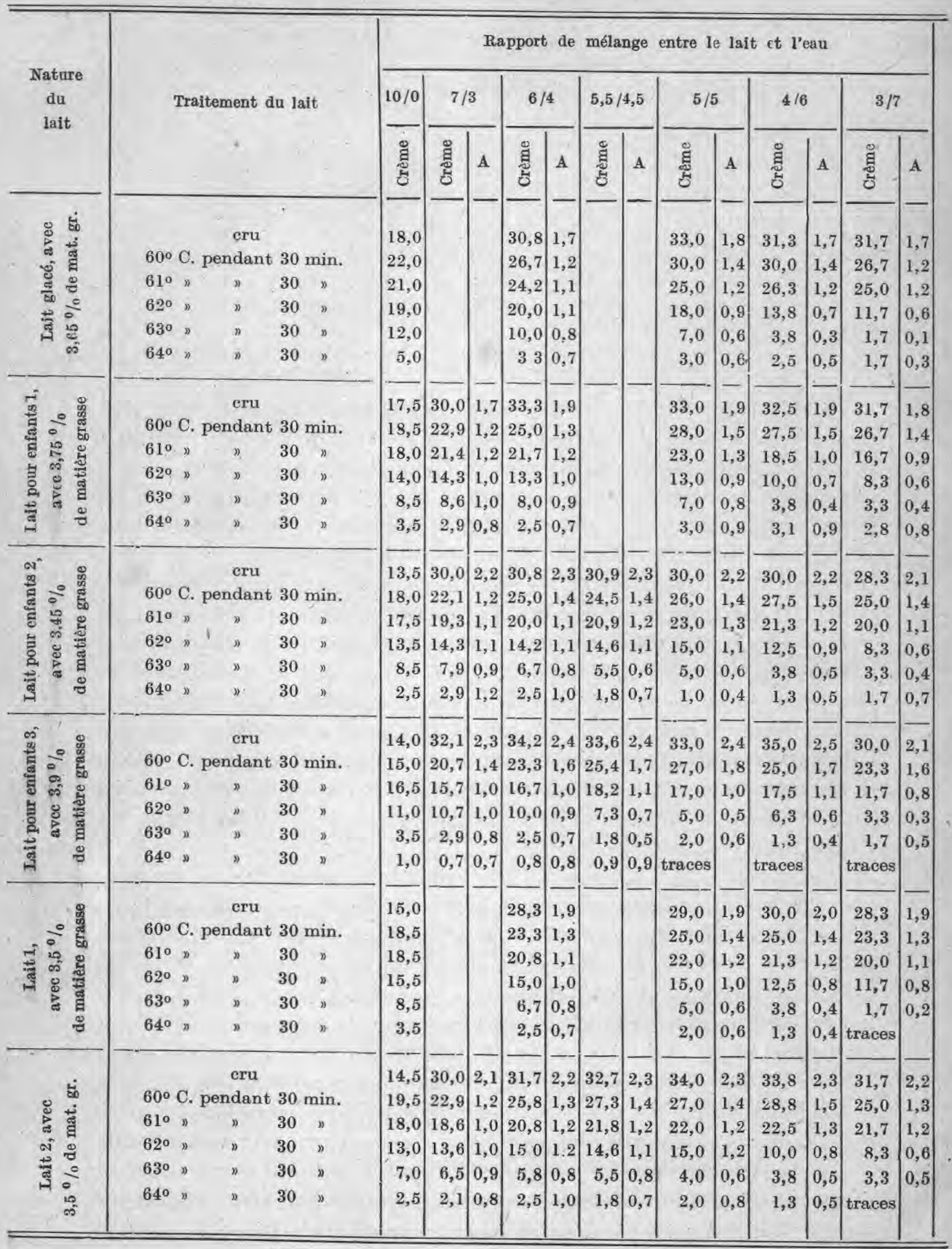


TABLEAU XXXVII

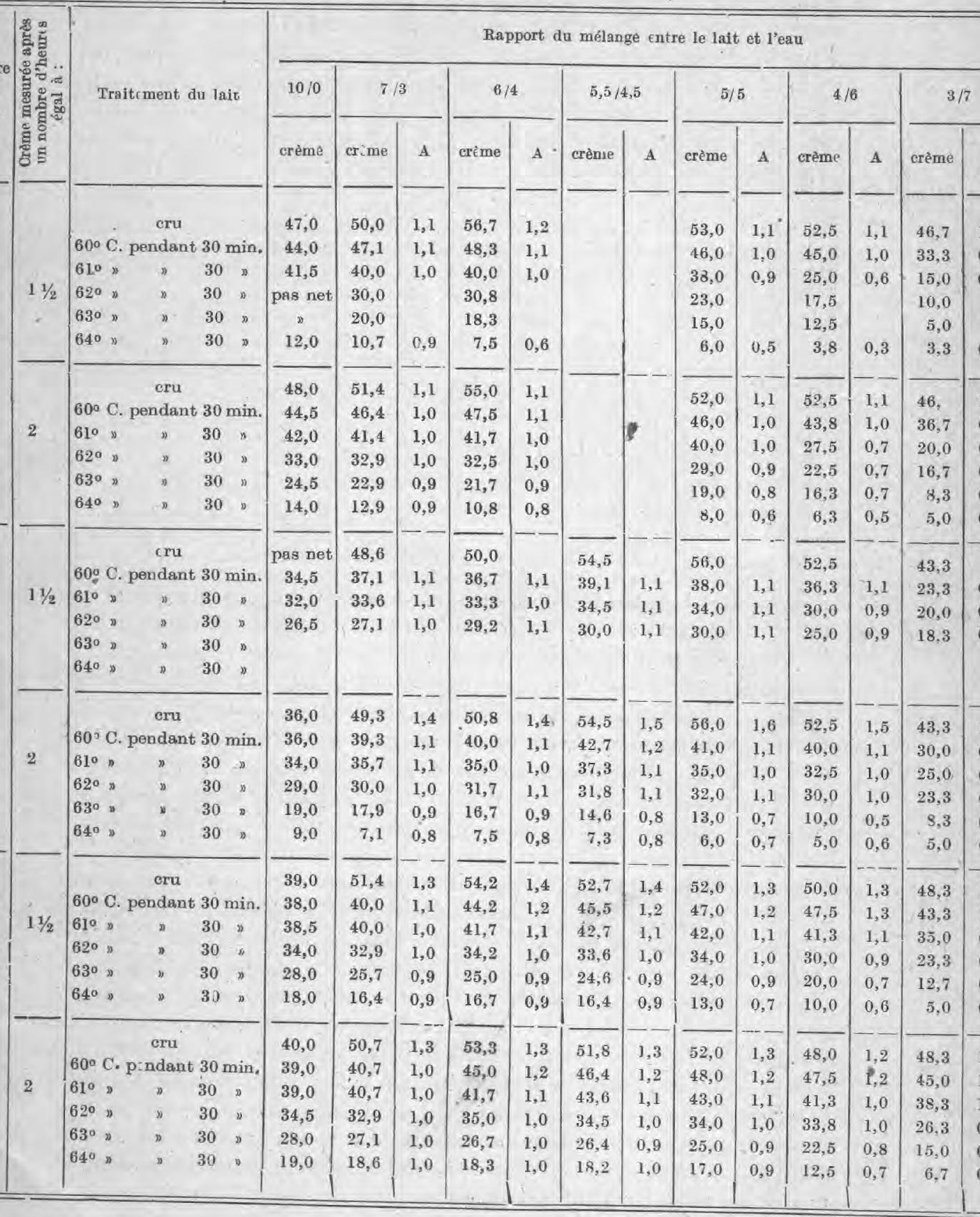


habituellement employé ; parcontre, en ce qui conzerne les laits de Jerseyaise 1 et 3 , il est près du rapport de mélánge $6 / 4$ sans que ceci ait une importance telle que cela soit prépondérant dans le rapport $\mathrm{A}$, qu'il est désirable d'obtenir aussi grand que possible en ce qui concerne le lait cru.

En ce qui concerne le lait chauffé il se montre exact que A descende au-dessous de 1 à des températures un peu plus élevées quand nous étendons un pəu moins le lait. Pour plus de clarté nous avons rassemblé les résultats des 2 présédents tableaux dans le tableau XXXVIII.

TABLEAU XXXVIII

\begin{tabular}{|c|c|c|c|c|c|c|c|c|c|}
\hline \multirow{2}{*}{$\begin{array}{c}\text { Rapport } \\
\text { de mélange } \\
\text { entre le lait } \\
\text { et l'eau }\end{array}$} & $\begin{array}{l}\text { Lait } \\
\text { glacé }\end{array}$ & $\begin{array}{c}\text { Lait } \\
\text { pour } \\
\text { enfants } \\
I\end{array}$ & $\mid \begin{array}{c}\text { J.ait } \\
\text { pour } \\
\text { enfants } \\
2\end{array}$ & $\begin{array}{c}\text { Lait } \\
\text { pour } \\
\text { enfants } \\
3\end{array}$ & $\begin{array}{l}\text { Lait } \\
\text { I }\end{array}$ & $\underset{2}{\text { Lait }}$ & $\begin{array}{c}\text { Lait de } \\
\text { Jer- } \\
\text { seyai:e } \\
\text { I }\end{array}$ & $\begin{array}{c}\text { Lait de } \\
\text { Jer- } \\
\text { seyaise } \\
2\end{array} \mid$ & Lait de Jerseyaise \\
\hline & \multicolumn{9}{|c|}{$\begin{array}{l}\text { Le rapport } A \text { descend au-dessous de I quand le lait a été chauffé à degrés C. } \\
\text { (crème mesurée après deux teures) }\end{array}$} \\
\hline $7 / 3$ & & 64 & 63 & 63 & & 63 & 63 & 63 & an-dessus de 64 \\
\hline $6 / 4$ & 63 & 63 & 63 & 62 & 63 & 63 & 63 & 63 & au-dessus de $6 t$ \\
\hline $5 / 5$ & 62 & 62 & 63 & 62 & 63 & 63 & 62 & 63 & 63 \\
\hline $4 / 6$ & 62 & 62 & 62 & 62 & 62 & 62 & 61 & 63 & 63 \\
\hline $3 / 7$ & 62 & 61 & 62 & 61 & 62 & 62 & 60 & 60 & 62 \\
\hline
\end{tabular}

D'après ce tableau, le changement du rapport A est plus fréquemment à $63^{\circ} \mathrm{C}$. quand on mélange 3 parties de lait avec 2 parties d'eau que quand on mélarge ensemble autant d'eau que de lait, et pourtant nous avons, dans cette suite d'essais, eu plus que d'habitude des échantillons de lait où $\mathrm{A}$ avec notre rapport de mélange habituel change à $63^{\circ} \mathrm{C}$. Avec ce rapport de mélange, $\mathrm{A}$ change dans la piupart des cas déjà à $62^{\circ} \mathrm{O}$. Il y a dons beaucoul de chanses pour que notre réaction devienne plus nette quand on mélange 3 lparties de lait avec 2 parties d'eau. Ceci est toutefois une question pour laquelle la pratique doit décider. Il ne faut pas oublier que quand il s'agit précisément de pasteuriser à aussi basse température que possible, il ne faut pas rendre le contrôle tellement sévère qu'on oblige les établissements laitiers à pasteuriser à $64^{\circ} \mathrm{C}$. quand il a été prouvé que $63^{\circ} \mathrm{C}$. sont suffisants. Comme il est défendu au Danemark de .vendre du lait pasteurisé à basse température, nous n'avons pas eu l'occasion d'étudier la question dans la pratique.

Nous venons de voir que l'agglutinine, qui est le facteur principal de la montée de la crème, passe en grande partie dans le sérum du lait. Le dernier point dans notre tentative d'éclaircir le problème de la montée de la crème sera ensuite de montrer si l'agglutinine du lait, comme celle du sérum du sang, suit la fraction de globuline, ce qui a ppuiera la supposition par avance naturelle que ces agglutinines sont identiques comme le sont les globulines du lait et du sérum. En vérité, nous devrions 
montrer que l'agglutinine a suivi la fraction d'euglobuline, mais comme au plus, $1 \%$ des matières albuminoïdes du lait est de la globuline, on comprendra facilement qu'il est impossible de vouloir séparer l'euglobuline et la pseudoglobuline. II est aussi très difficile d'obtenir une fraction de globuline exempte d'albumine, mais ce n'est du reste pas da tout nécessaire pour le but que nous cherchons, car si on peut seulement montrer que la montée de la crème dans le lait chauffé est améliorée d'autant plus vigoureusement par l'addition de lactoglobuline que cette lactoglobuline est exempte d'albumine, c'est tout ce dont nous avons besoin.

Pour la préparation de la lactoglobuline, le sérum acide est à préférer au sérum à la présure parce qu'il est exempt de produits provenant de la brisure de la caséine. Par neutralisation du sérum acide jusqu'à obtention de la concentration normale en ions $\mathrm{H}$ du lait et saturation avec du sulfate de magnésie à $30^{\circ} \mathrm{C}$. on obtient la séparation de toute la globuline avec une partie de l'albumine et en ajoutant une quantité convenable d'acide (environ $1 \%$ d'acide acétique) au filtrat, on en sépare le reste de l'albumine. Comme, ainsi que nous l'avons vu, même de petites quantités de sel agissent très vigoureusement pour arrêter la montée de la erème, il est nécessaire de soumettre ces fractions à une dialyse très attentive, pour laquelle nous avons employé la méthode du laboratoire de CARLSBERG (1).

Avec l'aide des déterminations d'azote, on peut se rendre compte de la globuline et de l'albumine originales du lait que nous avons dans nos fractions. Dans notre premier essai, alors que la caséine avait été séparée du lait par l'acide acétique, le sérum acide ainsi formé ne fut pas du tout neutralisé et le résultat fut que pratiquement toute l'albumine alla dans la fraction de globuline $\left(\mathrm{G}_{1}\right)$. Dans 2 autres préparations de globuline $\left(\mathrm{G}_{2}\right.$ et $\mathrm{G}_{3}$ ) dans lesquelles nous avons neutralisé le sérum jusqu'à ce qu'il ait atteint la concentration normale en ions $\mathrm{H}$ du lait, la fraction de globuline représentait environ $1 / 3$ du total globuline + albumine. Si toutefois l'agglutinine suit la globuline, $\mathrm{G}_{2}$ et $\mathrm{G}_{3}$ devraient donc contenir 3 fois plus d'agglutinine que $\mathrm{G}_{\mathbf{1}}$. Le lait et le sérum acide contiennent en moyenne $0,4 \%$ de globuline $\uparrow$ albumine. Ainsi que nous l'avons vu dans le tableau XXXI, la moitié seulement environ de l'agglutinine du lait passe dans le sérum; on aurait dû, par suite, pouvoir redomer au lait chauffé sa faculté originale de montée de la crème en l'additionnant de $0,8 \%$ de $\mathrm{G}_{1}$ ou de $0,27 \%$ de $\mathrm{G}_{2}$ ou de $\mathrm{G}_{3}$. Nos essais montrent cependant que le calcul n'est pas aussi simple, ear les fractions de globuline possèdent outre la propriété attendue de montée de la erème un effet retardateur de cette montée qui, comme les essais de contrôle nous l'ont montré, ne peut pas être attribué aux traces de sulfate de magné-

(1) S. P. L. Sorensen : Etudes sur les protéines (Communication du Laboratoire de Carlsberg, 1917, XII, p. 24). 
sium qui l'accompagrent. L'effet retardateur fut le plus grand dans la préparation $\mathrm{G}_{\mathbf{1}}$ riche en albumine, et pour de grandes doses de cette préparation, son action retardatrice pouvait devenir plus grande que l'action favorable. Seule la préparation $\mathrm{G}_{2}$ agit d'une façon tout à fait satisfaisante.

Les fractions d'albumine eurent une action retardatrice sur la montée de la crème analogue à celle des fractions de globuline, mais, au contraire de celles-ci, ne montrèrent pas trace d'action favorable, ce qui prouve que l'agglutinine, dans le lait comme dans le sang, suit la globuline.

Comme nous avons nos fractions d'albumine en solution, nous ne pouvons dans ces essais travailler qu'avec du lait étendu, et comme nous étendons continuellement avec les mêmes quantités de solution d'albumine, son pourcentage en albumine devient précisément le pourcentage qui est apporté au lait. Pour nos premiers essais qui sont indiqués dans le tableau XXXIX nous avons employé une solution avec $0,33 \% \mathbf{G}_{\mathbf{1}}$.

TABLEAU XXXIX

\begin{tabular}{|c|c|c|c|c|c|c|c|c|c|c|}
\hline \multirow{3}{*}{\multicolumn{5}{|c|}{ Traitement du lait }} & \multicolumn{2}{|c|}{ Lait I } & \multicolumn{2}{|c|}{ Lait $2 a$} & \multicolumn{2}{|c|}{ Lait $2 \mathrm{~b}$} \\
\hline & & & & & \multicolumn{6}{|c|}{ Globuline } \\
\hline & & & & & o & + & o & + & o & + \\
\hline \multicolumn{4}{|c|}{ oru } & & 32,0 & 28,0 & 24,0 & 16,0 & 1,0 & traces \\
\hline \multicolumn{5}{|c|}{$63^{\circ} \mathrm{C}$. pendant $30 \mathrm{~min}}$. & 4,0 & 12,0 & 5,0 & 7,0 & traces & traces \\
\hline $68^{\circ}$ & » & 5 & » & . & traces & 10,0 & 1,0 & 4,0 & traces & traces \\
\hline $70^{\circ}$ & $n$ & 5 & " & & & & traces & 3,0 & traces & traces \\
\hline $75^{\circ}$ & " & 5 & x & & & & traces & 1,0 & traces & traces \\
\hline $80^{\circ}$ & " & 2 & $n$ & & traces & 20,0 & traces & 21,0 & traces & 18,0 \\
\hline
\end{tabular}

Si nous ne regardons provisoirement que le lait 1 et le lait 2 a, nous voyons que la globuline dans le lait cru n'a qu'une action retardatrice sur la montée de la crème. Comme on peut admettre que le lait cru contient à l'avance un excès d'agglutinine, il est à peine possible de pouvoir améliorer la montée de la crème dans le lait cru par addition de globuline. Dans le lait chauffé, la propriété qu'a la globuline de former de la crème est par contre suffisamment manifeste ; mais il se passe ici tout à fait ce qui se passe quand nous additionnons du lait cru à du lait chauffé : l'action pour une quantité déterminée est d'autant plus faible que le chauffage a été plus fort, jusqu' ‘ ce qu'on atteigne le point auquel la séparation de la crème est aidée par la coagulation de l'albumine. A cet égard le lait $2 \mathrm{~b}$ donne un exemple amusant. $2 \mathrm{~b}$ est le même lait que $2 \mathrm{a}$ (qui a $3,8 \%$ de crème), mais nous y avons détruit la faculté de montée de la crème en l'additionnant de $1,7 \%$ de crème homogénéisée (de telle 
sorte que $31 \%$ des globules de matière grasse du lait étaient homogénéisés). Ici, aussitôt que la coagulation de l'albumine peut aider l'action de l'agglutinine, ce lait laisse aussi monter la crème.

TABLEAU XL

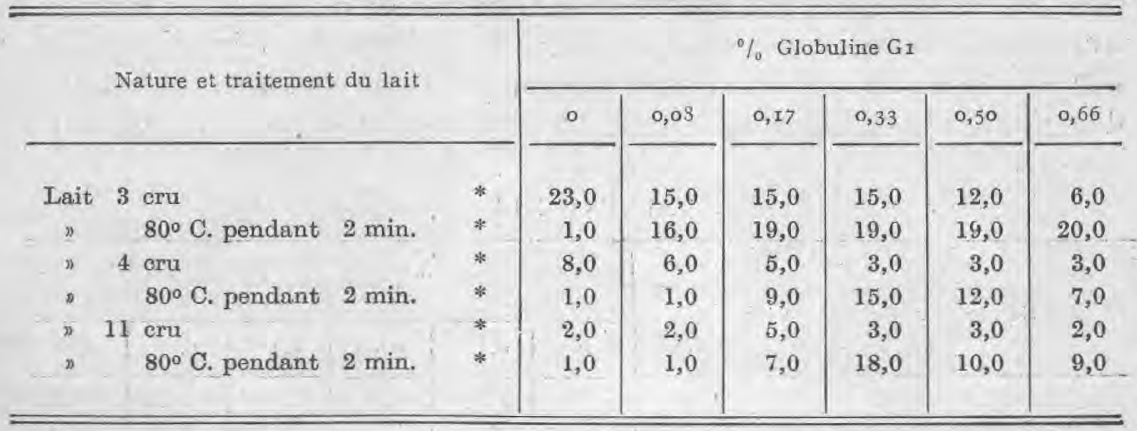

Le tableau XL montre comment diverses proportions de globuline $\mathrm{G}_{1}$ agissent sur la montée de la crème dans 3 échantillons de lait différents. Dans le lait cru, la montée de la crème diminue quand la proportion de globuline augmente. Le lait 11 constitue toutefois une exception, car la montés de la crème atteint son maximum avec $0,17 \%$ de globuline. Ce lait est toutefois identique au lait 11 du tableau IX que, pour des raisons diverses, nous soupçonnions d'avoir subi un léger chauffage, et nous voyons maintenant d'une façon tout à fait différente que nos soupçons sont confirmés. Quand le lait a été chauffé 2 minutes à $80^{\circ} \mathrm{C}$., les laits 4 et 11 montrent un optimum net précisément a vec la proportion $\mathrm{G}_{1}(0,33 \%)$ que nous avons employée dans nos premiers essais. Par contre, le lait 3 semble, après avoir été chauffé, être moins sous l'influence de la proportion de globuline, et donne déjà une crème abondante avec seulement $0,08 \% \mathrm{G}_{1}$. Malheureusement, on ne peut pas comparer les couches de crème dans les laits chauffés à $80^{\circ} \mathrm{C}$. avec les couches de crème dans les laits crus, parce que les premiers contiennent de l'albumine.

Aussitôt que les fractions de globuline isolées, ou seulement après avoir été mélangáes avee du lait, sont chauffées 5 minutes à $70^{\circ} \mathrm{C}$., elles ne produisent plus trace de montée de crème parce que.toute l'agglutinine qu'elles contenaient a été détruite.

Le tableau XLI montre que la préparation $\mathrm{G}_{2}$, à l'encontre de $\mathrm{G}_{1}$, est pratiquement exempte de produits retardateurs. Les différences que le lait cru montre dans la quantité de crème lors de l'addition de diverses proportions de globuline, sont inférieures aux erreurs possibles. La préparation est si forte que le lait ayant subi la pasteurisation lente retrouve sa fasulté de montés de crème entière avec environ $0,1 \%$ de cette préparation. Dans le lait un peu plus ehauffé, nous n'avons pas, par contre, la 
faculté de montée de crème entièrement régénérée, même si on ajoute $0,5 \% \mathrm{G}_{2}$. Ceci pouvait signifier que l'agglutinine n'est pas le seul facteur thermolabile de la montée de la crème, mais qu'il peut se trouver un autre facteur dans le lait (qui probablement n'est détruit qu'à une température plus élevée que l'agglutinine), car sans cela on devrait aussi obtenir une montée de crème tout à fait complète dans le lait chauffé au-dessus de $63^{\circ}$ C., quand on l'additionne seulement d'une quantité suffisante d'agglutinine sous la forme d'une préparation aussi peu retardatrice que $\mathrm{G}_{2}$.

TABLEAU XLI

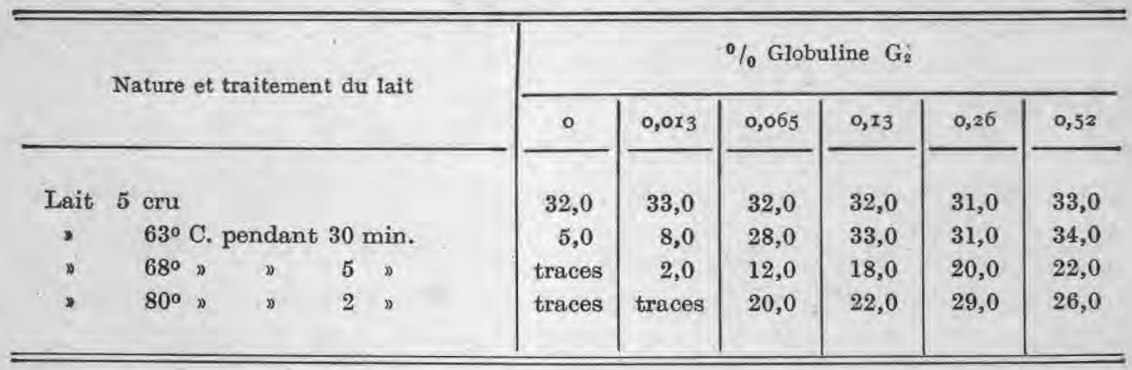

Pour élucider cette question, nous avons mélangé du lait écrémé, qui était cru, ou qui avait été chauffé, a vec de l'eau ou avec une solution à $0,1 \%$ de la préparation de globuline $\mathrm{G}_{3}$, dans les conditions indiquées plus en détail dans le tableau XLII. Pour éviter l'influence qu'une teneur plus ou moins grande en matière grasse peut exercer, celle-ci fut réglée dans tous les mélanges, grâce à l'addition de crème concentrée, soit à $2,5 \%$, soit à $5 \%$ de matière grasse. Ceci fut exécuté en donnant dès le début ces proportions de matière grasse aux trois composants. Dans ce cas, c'est la couche de crème mesurée directement qui est notée ; nous ne l'avons pas, comme c'était le cas quand on travaille avec du lait étendu, multipliée par le facteur de dilution.

Si nous regardons d'abord les mélanges où le lait est cru, on voit que la montée de la crème diminue beaucoup avec les deux plus petites proportions de lait, et ceci à peu près aussi fortement, que nous ayons étendu avec de l'eau ou a vec de la solution de globuline. D'autres essais nous ont aussi montré que le mélange crème + eau, exempt de lait, ne peut pas laisser monter de la crème uniquement à l'aide de l'agglutinine ; pour cela, il faut, en outre, une petite quantité de lait cru ou de sérum cru.

Si nous regardons ensuite les mélanges où le lait a été chauffé, ce n'est naturellement qu'a vec la solution de globuline qu'ils laissent monter des quantités appréciables de crème, mais ici aussi la couche de crème devient plus petite au fur et à mesure que la proportion de lait diminue, et que la proportion d'agglutinine augmente. En ce qui concerne le lait qui a été chauffé à $80^{\circ} \mathrm{C}$, , ceci peut être en rapport avec ce que la pro- 
TABLEAU XLII

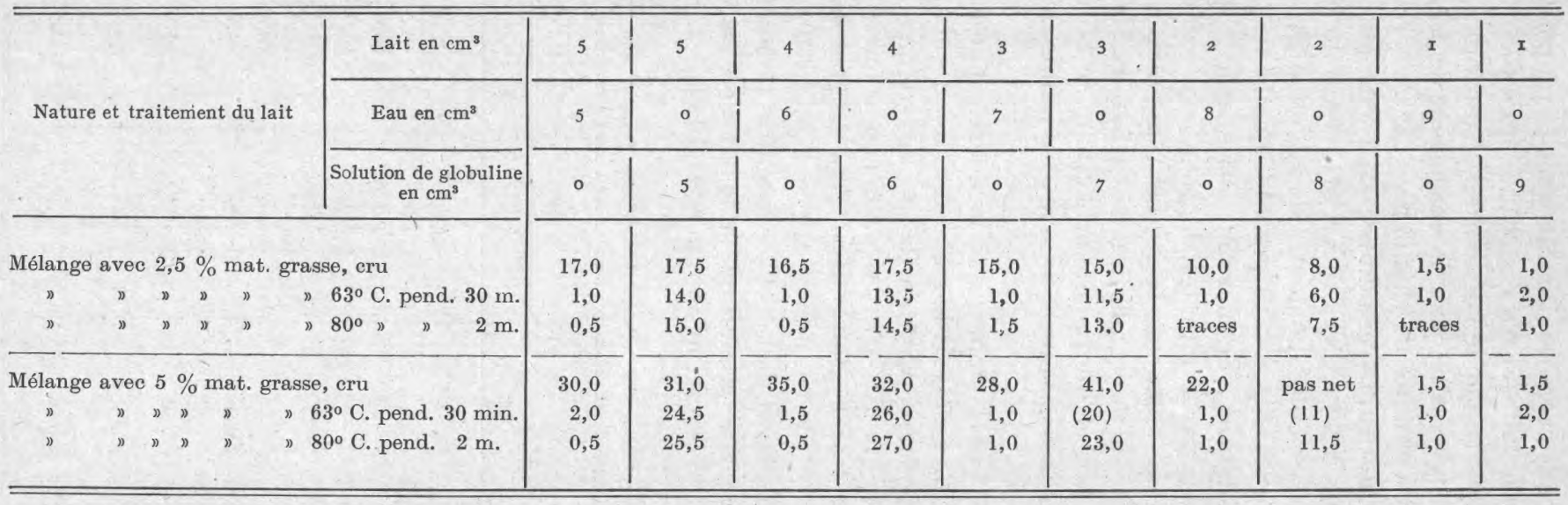


portion d'albumine coagulée diminue avec la proportion de lait, mais en ce qui concerne le lait qui n'a été chauffé qu'à $63^{\circ} \mathrm{C}$., l'explication doit être cherchée dans ce que le lait, outre l'agglutinine, contient encore un facteur qui est nécessaire pour la formation de la crème, facteur qui supporte donc 30 minutes de chauffage à $63^{\circ} \mathrm{C}$.

Nos essais antérieurs avec addition de 1/5 de lait cru au lait qui a été chauffé (tableaux XXV, XXVI et XXVII) montrent que le lait atteint un minimum dans la faculté de montée de la crème par un court chauffage à $75^{\circ}-77^{\circ} 5 \mathrm{C}$., et il est par suite probable que l'autre facteur créateur de crème est déjà détruit à ce moment. Par chauffage plus prolongé à ces températures, ou par 2 minutes de chauffage à $80^{\circ} \mathrm{C}$., la faculté de montée de la crème augmente bien à nouveau, mais c'est, comme nous le savons, par suite de la coagulation de l'albumine; [mais la question ne devient tout à fait claire que quand l'albumine est de nouveau soluble à $95^{\circ}-97^{\circ} 5 \mathrm{C}$., et nous avons là un nouveau minimum pour la montée de la crème.

Le tableaụ XLIII montre que la même chose se répète quand, an: lieu de lait cru, on ajoute de la globuline au lait qui a été chaufté. L'essai fut exécuté avec la préparation G3 qui malgré qu'elle ne contenait pas plus d'albumine que $\mathrm{G} 2$, n'a pas pu, même à fortes doses, redonner au lait, qui avait été chauffé à $63^{\circ} \mathrm{C}$., sa faculté de montée de crème originale.

Nous voyons aussi dans cet essai que le premier minimum pour la montée de la crème se produit quand le lait a été chauffé instantanément à $75^{\circ}-80^{\circ} \mathrm{C}$., et le second minimum quand le lait a été chauffé à $97^{\circ} 5 \mathrm{C}$.

TABLEAU XLIII

\begin{tabular}{|c|c|c|c|c|}
\hline \multirow{2}{*}{\multicolumn{2}{|c|}{$\begin{array}{c}\text { Lait four enfants } \mathrm{I} \text {, avec } 3,75 \% \mathrm{~m} . \mathrm{gr} \text {. } \\
\text { Traitement }\end{array}$}} & \multicolumn{3}{|c|}{${ }^{0}$ Globuline $\mathrm{G}_{3}$} \\
\hline & & o & 0,25 & $x, 0$ \\
\hline & cru & 36,0 & 37,0 & 32,0 \\
\hline $63^{\circ}$ & C. pendant $30 \mathrm{~min}$. & 4,0 & 12,0 & 20,0 \\
\hline $68^{\circ}$ & $n \quad n \quad 5 n$ & 1,0 & 10,0 & 18,0 \\
\hline $70^{\circ}$ & " instantané & 8,0 & 16.0 & 20,0 \\
\hline " & " pendant 2 min. & 1,0 & 11,0 & 20,0 \\
\hline$"$ & $\Rightarrow \quad 50$ & traces & 10,0 & 19,0 \\
\hline $75^{\circ}$ & " instantané & traces & 6,0 & 20,0 \\
\hline$"$ & pendant 2 min. & traces & 4.0 & 21,0 \\
\hline $77^{\circ} 5$ & . instantané & traces & 4,0 & 21,0 \\
\hline$"$ & pendant $2 \mathrm{~min}$. & traces & 4,0 & 23,0 \\
\hline $80^{\circ}$ & ఎ instantané & traces & 3,0 & 22,0 \\
\hline $n$ & pendant $2 \mathrm{~min}$. & traces & 12,0 & 22,0 \\
\hline $97^{\circ} 5$ & " instantané & 1 & 1,0 & 1,0 \\
\hline$" x$ & "pendant $2 \mathrm{~min}$. & 2 & 1,0 & 1,0 \\
\hline
\end{tabular}


Au premier minimum toutefois, la montée de crème se laisse régénérer si seulement on ajoute suffisamment d'agglutinine $(1 \% G)$, tandis que ee n'est plus possible lors du second minimum. On ne peut donc pas mettre en doute que lors de la montée normale de crème dans le lait cru, outre l'agglutinine, un autre facteur thermolabile agit, qui ne passe pas dans la fraction de globuline, et qui n'est détruit qu'à $75^{\circ} \mathrm{C}$., et peut-être seulement entièrement à $97^{\circ} 5 \mathrm{C}$.

Pour élucider ce point, nous avons ajouté à de l'eau, d'une part $0,25 \%$ de solution de globuline $(10 \%$ d'une solution à $2,5 \%$ de globuline) et d'autre part des proportions diverses de sérum de lait chauffé à diverses températures. Par addition de crème concentrée, tous les mélanges furent amenés à une proportion de matière grasse de $4 \%$. Le tableau XLIV indique les proportions de crème séparées dans ces mélanges.

TABLEAU XLIV

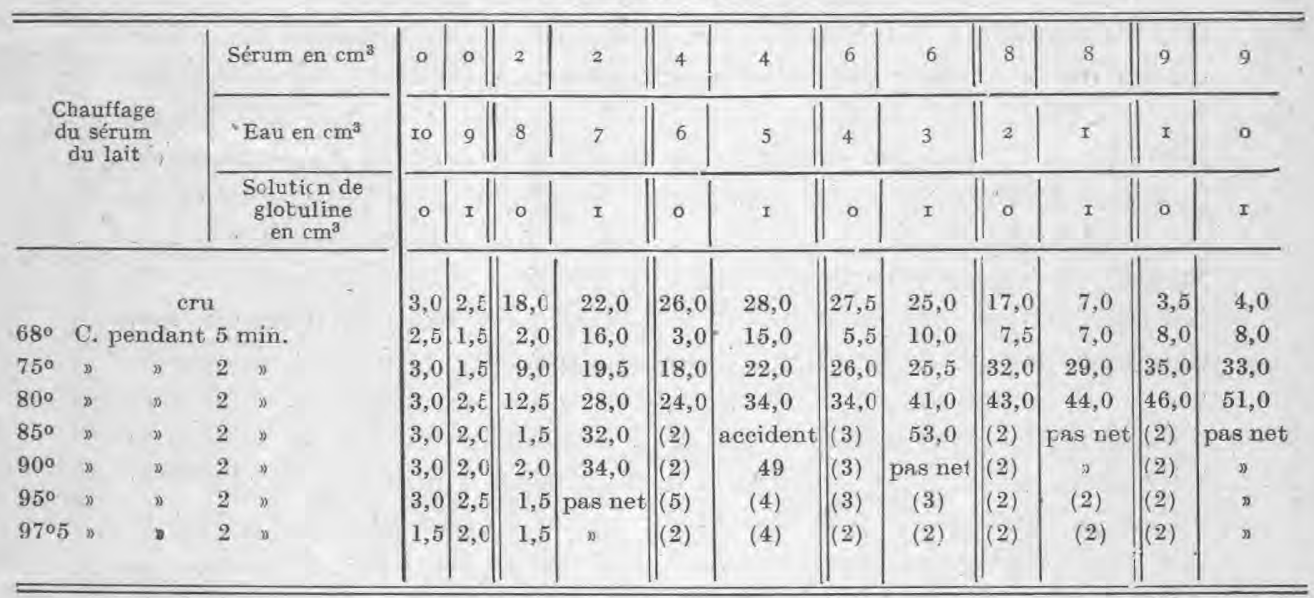

Comme les chiffres des 2 premières colonnes du tableau sont pratiquement identiques, on voit que l'agglutinine seule ne produit aucune montée de crème dans l'eau; ce n'est que quand, en outre, on ajoute $1 / 5$ de sérum de lait qu'on obtient la montée de crème, pour autant que ce sérum n'ait pas été chauffé plus de 2 minutes à $90^{\circ} \mathrm{C}$. Si, par contre, le sérum a été chauffé 2 minutes à $95^{\circ} \mathrm{C}$., la montée de la crème est imprécise et seulement très faible. Ce n'est qu'à cette température qu'est détruit par conséquent le second facteur thermolabile, tandis qu'ici l'agglutinine a déjà été détruite, comme dans tous nos précédents essais, après 5 minutes de chauffage à $68^{\circ} \mathrm{C}$. Les résultats de l'essai deviennent moins clairs, lorsqu'on emploie de grandes quantités de sérum, car plus grande est la proportion d'albumine coagulée qui facilite aussi la montée de la crème, et si $9 / 10$ du mélange sont du sérum, nous obtenons le 
résultat remarquable qu'on obtient plus de crème séparée lorsque le sérum a été chauffé 5 minutes à $68^{\circ} \mathrm{C}$. que quand il est cru. En effet, dans le sérum cru, presque non étendu, il n'y a, comme nous l'avons montré précédemment, qu'une très petite montée de crème par suite de la forte concentration saline. A $68^{\circ}$ C., il y a toutefois suffisamment d'albumine coagulée pour que cela facilite la montée de la crème. Du second facteur thermolabile nécessaire à la montée de la crème, nous ne savons que ce qui suit, e'est-à-dire qu'il passe dans le sérum, mais qu'il n'est présipité ni avec la globuline ni avec l'albumine. Comme l'agglutination des globules du lait exige un complément, cette action est plutôt une conglutination.

Mentionnons ici que le colostrum qui contient beaucoup de globuline, contient aussi 5 à 10 fois plus d'agglutinine que le lait ordinaire ; l'addition seulement de $1 \%$ de colostrum améliore la montée de la crème dans le lait chauffé.

Avant que nous abandonnions le chapitre de la montée de la crème, il est naturel que nous eherchions à répondre à la question de savoir quelle est sa valeur quand il s'agit de lait pour des usages domestiques.

Nous pouvons faire abstraction de ce que beaucoup de mères de famille appréoient la teneur en graisse du lait d'après l'épaisseur de la couche de crème qu'il forme en un certain temps, car quelques renseignements à ce sujet leur apprendront vite que ceci n'est exact que pour le lait fraîchement trait, et non pour le lait qui a été agité, refroidi, ou pasteurisé. Si la mère de famille ne désire pas précisément obtenir elle-même un peu de crème avec le lait acheté, il est plus pratique pour le ménage d'avoir un lait qui ne laisse pas trop facilement monter sa crème, do telle sorte qu'on n'ait pas besoin de le remuer à nouveau, chaque fois qu'on en emploie un pou. Cet inconvénient s'évite en pasteurisant le lait par chauffagə instantané à $75^{\circ} \mathrm{C}$. ou par un chauffage de 5 minutes à $68^{\circ} \mathrm{C}$., prozédés qui tous deux se laissent exésuter d'une façon continue. Grâce à la destruction de la faculté de montée de la crème, il est facile de contrôler si le lait a été chauffé aussi fortement. Pour prouver si le lait a été chauffé au-dessus de cette limite, on n'a toutefois que la réaction de STORCH, tandis que, eomme limite supérieure pour le lait ayant été soumis à la pasteurisation lente, on a la destruction de la faculté de montée de la crème qui dans les méthodes précédemment indiquées était employée comme limite inférieure.

Si cependant on considère la question au point de vue de la physiologie alimentaire, il s'élève un doute sur la question de savoir s'il est bon de pasteuriser le lait tellement fortement qu'on détruise sa faculté de montés de crème. Par elle-même, cette propriété n'a naturellement pas d'importanze physiologique, puisque le lait est destiné à l'allaitement au sein, mais les agglutinines qui produisent la montée de la crème ont sûre- 
ment un certain but. Nous savons que, dans le colostrum, se trouvent, outre des antitoxines, des agglutinines qui quand elles passent avec la globuline dans le sang des jeunes animaux, les protègent contre beaucoup de maladies. Le lait de vache n'est certainement destiné qu'aux veaux, et il est par conséquent possible que les produits immunisants qui y sont contenus soient tout à fait sans importance pour les enfants qui, d'ailleurs, à l'encontre des veaux, déjà avant leur naissance reçoivent les immunisines de leur mère. Il me semble cependant qu'avant que la nature des agglutinines formatrices de crème soit plus amplement fixée, on doit se garder de chauffer le lait qui doit être employé à la nourriture des enfants tellement fortement que ces substances soient entièrement détruites. Jusqu'à nouvel ordre, la pasteurisation lente peut donc être consdiérée comme étant la meilleure méthode de pasteurisation basse du lait.

(A suivre)

\title{
LA PRODUCTION DU LAIT DE CONSOMIMATION EN DANEMARK
}

\author{
par J. de GIBON \\ Ingénieur Agronome \\ GÉNÉRALITÉS.
}

\section{Article Premier. - Le problème du latt a Copenhagne.}

Il ne saurait être question dans le cadre de cette étude d'étudier le problème du lait destiné à la consommation dans toute l'étendue du fays danois.

L'exemple du ravitaillement en lait à Copenhague sera seul envisa gé.

Les 730.000 habitants de Copenhague consomment journellement environ 275.000 litres de lait soit une moyenne de 4/10 de litre par habitant et par jour.

Un recensement de 1925 relève qu'en juillet de la même année, ce lait était fourni par 58.787 vaches appartenant à 3.610 étables.

Les 13.000 litres de lait pour enfants étaient produits par 2.000 vaches (19 étables) et les 3.500 litres de lait de Jersey par 1.000 vaches environ (10 étables).

$85 \%$ du lait venàu à Copenhague était soumis à un contrôle qualitatif. Ce dernier lajt était fourni en grande majorité par les cultivateurs fournisseurs des grandes laiteries, le reste par les laiteries coopératives de la campagne, dans la proportion de $85 \%$ à $15 \%$.

Les producteurs de lait se sont réunis en sociétés locales, fédérées elles-mêmes en un organisme central, pour la défense de leurs intérêts. lait.

3 grandes laiteries assurent à elles seules $85 \%$ du ravitaillement en

Ce sont deux sociétés privées, sociétés par actions : 